\title{
STEREOSCOPIC VIEWING OF DIGITAL HOLOGRAMS OF REAL-WORLD OBJECTS
}

\author{
Taina M. Lehtimäki ${ }^{l}$ and Thomas J. Naughton ${ }^{1,2}$ \\ ${ }^{1)}$ University of Oulu, RFMedia Laboratory, Oulu Southern Institute, Vierimaantie 5, 84100 Ylivieska, \\ Finland. Email: firstname.lastname@oulu.fi \\ 2) Department of Computer Science, National University of Ireland, Maynooth, County Kildare, Ireland
}

\begin{abstract}
We have studied the use of conventional stereoscopic displays for the viewing of digital holograms of real-world three-dimensional (3D) objects captured using phase-shift interferometry. Although digital propagation of holograms can be performed efficiently, only one depth-plane of the scene is in focus in each reconstruction. Reconstruction at every depth to create an extended-focus image is a timeconsuming process. We investigate the human visual system's ability to perceive 3D objects in the presence of blurring when different depth reconstructions are presented to each eye. Our digital holograms are sufficiently large that subregions can be digitally propagated to generate the necessary stereo disparity. The holograms also encode sufficient depth information to produce parallax. We find that our approach allows 3D perception of objects encoded in digital holograms with significantly reduced reconstruction computation time compared to extended focus image creation.
\end{abstract}

Index Terms - Signal processing for diffraction and holographic 3DTV, Stereoscopic display techniques, Holographic display technology, Human factors, Stereo vision, Three-dimensional displays, Image processing, Holographic recording

\section{INTRODUCTION}

Holograms are a natural way of encoding three-dimensional (3D) information about a real-world scene [1]. Digital holograms [2-6], created using a laser and a digital camera, record the intensity and phase of the light scattered from the 3D object(s). These digital holograms contain 3D data, which are currently visualised with two-dimensional (2D) reconstructions from different viewing angles and with different in-focus depths. The challenge is how to be able to show the 3D features of the objects encoded in these digital holograms to the viewer. Few current 3D display technologies support digital holographic data and 2D reconstructions are just not enough for the viewer to easily perceive, understand, and appreciate the 3D scene. Some work has been done to extract 3D information from digital holograms in such a way that in theory it could be passed to a conventional 3D display, but to date this has only proved successful for microscopic objects and very short propagation distances so that speckle is not a problem [7] or for zero-dimensional objects such as particles [8]. Optical displays comprising a laser and a spatial light modulator could be used $[9,10]$, but these are either also only capable of reconstructing a single 2D perspective themselves or are costly special-purpose devices. This means that, in general, 2D displays are the only option for viewing reconstructions from digital holograms. In this paper we propose a simple but effective way of visualising digital holograms with a consumer-level stereoscopic display.

The challenges in visualising digital holograms of realworld 3D objects are (1) large calculation times required for the reconstruction of different perspectives and different depths for large holograms, (2) the amount of noise (speckle and otherwise) present in the reconstructions [11], and (3) the fact that only one depth plane is in focus in each reconstruction given that good stereo perception requires high spatial frequencies (small amount of blurring) in the images $[12,13]$. The purpose of this study is to find out if is it possible to overcome these challenges and visualise digital holographic data in a way that allows people to use their natural stereoscopic 3D perception. In first part of our study we use digital holograms of objects with short depth which allows us to avoid the blurring effect in some parts of the reconstructions of the digital holograms. In the second part of our study we look further into the possibilities of combining a stereoscopic display with digital holograms to assess the limits of human perception with holograms encoding objects of greater depth.

\subsection{Human stereovision}

Stereovision is one principal way humans extract 3D information from a scene. Both eyes get slightly different images due to their different positions in the head. The human vision system is able to combine these different images forming a 3D perception of the scene: a process called stereopsis. It has been shown that typically the higher spatial frequencies are the most important for perception of 3D objects (meaning that stereo acuity declines when the amount of blurring increases) [13]. However, a study 
conducted by Papelba et al. [12] with disbalanced images suggests that within certain limits it is possible to perceive stimuli in $3 \mathrm{D}$ even when one eye is seeing a blurred image and one eye a focussed image.

In our study we also use disbalanced images, but due to the nature of the reconstructions of digital holograms, our stimuli are focussed for each eye at a different depth, since blurring in a digital hologram reconstruction corresponds to an incorrect depth reconstruction. For each stereo pair, we reconstruct two scenes from the digital hologram at different depths (for example one focussed at the front-most part of the scene and the other focused at the back-most part) and determine the quality of the stereo perception.

\subsection{Capturing the holograms}

Our digital holograms are recorded using the optical setup (shown in Fig. 1) based on a Mach-Zehnder interferometer architecture in an in-line configuration. A spatially filtered linearly polarized helium neon $(632.8 \mathrm{~nm})$ laser beam is split into object and reference beams, both of which are spatially filtered and expanded. The first beam illuminates the 3D object placed at a distance $d$ from a 10-bit $2032 \times 2048$ pixel CCD camera. The reference beam passes through half-wave plate $\mathrm{RP}_{1}$ and quarter-wave plate $\mathrm{RP}_{2}$. Through permutation of the fast and slow axes of the plates we can achieve phase shifts of $0,-\pi / 2,-\pi$, and $-3 \pi / 2$. The reference beam combines with the light diffracted from the object and forms an interference pattern in the plane of the camera. At each of the four phase shifts we record an interferogram. Using these four intensity images, the complex-valued camera-plane wavefront can be approximated in a computer to good accuracy using phaseshift interferometry [14,15]. Digital holograms of reasonably diffuse $3 \mathrm{D}$ objects were used. The objects had approximate dimensions of $5 \mathrm{~mm} \times 5 \mathrm{~mm} \times 5 \mathrm{~mm}$ and were positioned approximately $d=350 \mathrm{~mm}$ from the camera.

A perspective of the object encoded in the digital hologram is reconstructed by numerically simulating the propagation of light backwards in time and space. By propagating a distance $-d$ one reconstructs a perspective with all points of the object at distance $d$ in focus, and all other points out of focus proportional to their distance from $d$. By propagating the full hologram field one creates an onaxis perspective. By propagating a subset of pixels, and employing the appropriate linear phase tilt, one creates a (lower-resolution) perspective determined by the distance from the centre of this subset to the optical axis.

The range of viewing angles is determined by the ratio of the window size to the full CCD sensor dimensions. Our CCD sensor has approximate dimensions of $18.5 \mathrm{~mm} \times 18.5$ $\mathrm{mm}$ and so a $1024 \times 1024$ pixel window has a maximum lateral shift of $9 \mathrm{~mm}$ across the face of the sensor. With an

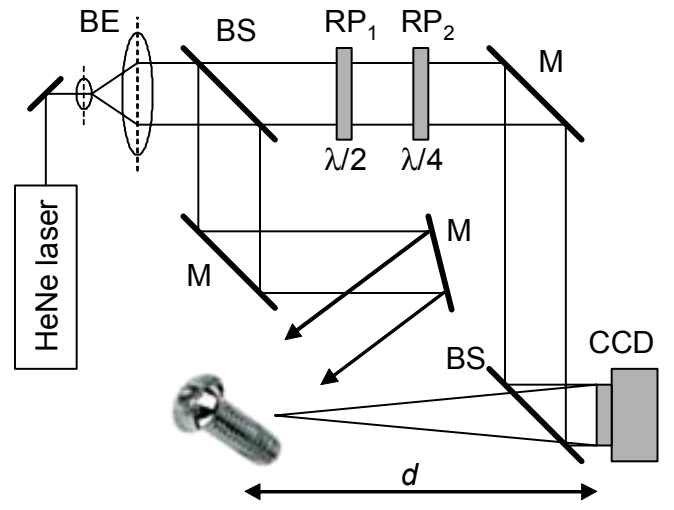

Figure 1: Experimental setup for hologram capture: BE, beam expander; BS, beam splitter; M, mirror; RP, retarder.

object positioned $d=350 \mathrm{~mm}$ from the camera, viewing angles in the range $\pm 0.74^{\circ}$ are permitted. Smaller windows will permit a larger range of viewing angles at the expense of image quality at each viewpoint.

\section{METHODS}

\subsection{Subjects and apparatus}

Our experiments were performed using six subjects. Their stereovision was tested with stereo pair photographs before the experiment to ensure they had good stereovision acuity. Three of the six subjects wore prescribed vision correction.

Stimuli were presented on a Sharp LL-151-3D LCD monitor. This is an autostereoscopic display; it allows users to see stereoscopic images without using special glasses. It consists of two LCD panels, one in front of another. In 3D mode the foreground LCD panel acts as a parallax barrier so that the right and left eyes of a viewer receive different images. The display resolution was $1024 \times 768$ pixels $(512 \times 768$ pixels for each eye) and the distance between the subject and display was approximately $60 \mathrm{~cm}$.

\subsection{Stimuli}

We used two types of stimuli to investigate if our subjects were able to see the stimuli in 3D. First we experimented with our idea of perception in 3D despite the noise in the reconstructions. For Experiment 1 we used digital holograms of objects with very small depth. With these holograms it was possible to create reconstructions at only one depth that still had the whole image appear largely infocus to the viewer. In Fig. 2 we show an example of our stereo pair stimuli of an object with small depth. Our stereo pairs consisted of reconstructions made with window sizes from 64 pixels to 1024 pixels. Mean filtering was applied to reduce speckle.

For Experiment 2 we used disbalanced stimuli reconstructed from a hologram of the two-object scene 
shown in Fig. 3 . We chose $512 \times 512$ pixel window size: a choice informed from the results of Experiment 1. In these stimuli, part of the image was blurred according to the reconstruction depth, as shown in Fig. 3. We used in total ten reconstructions (five different depths labelled $f, m f, m$, $m b$, and $b$ ranging from the front in focus $f$ to the back in focus $b$, for each of left and right perspectives). From these we assembled 9 stereo pairs, the labels of which are listed in Fig. 4, where $f b$ means in the left perspective the front of the scene was in focus and in the right perspective the back was in focus.

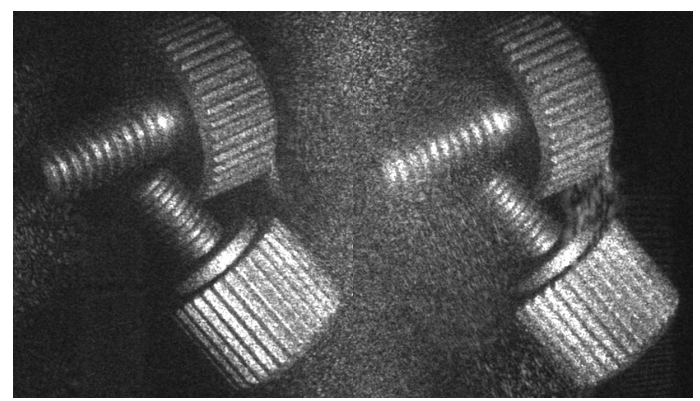

Figure 2: Left and right perspectives of a scene where almost all parts of the scene are in focus.

\subsection{Procedure}

In Experiment 1 we first asked subjects to grade the subjective quality of individual reconstructions presented on a conventional 2D display. Subjects were instructed to form their own opinion of high/low quality but to remain consistent throughout the experiments. They were asked to use a scale from 1 (very poor) to 9 (excellent). Next we asked them to grade stereo pairs presented on the autostereoscopic display according to the subjective quality of the stereo image and separately the amount of 3D effect perceived.

For Experiment 2 we first asked subjects to grade the amount of focus in the ten individual reconstructions for the front and back object separately. We then asked them to do the same for the nine stereo pairs presented on the autostereoscopic display.

\section{RESULTS}

- Despite the noise in the reconstructions and their general poor quality, consistent with all digital holograms using current technology, the stereo pairs were perceived in 3D by all test subjects.

- The quality of 3D perception and image quality depended on the reconstruction window size but only up to $512 \times 512$ pixel windows; likely due to our speckle reduction filtering.

- When a stereo pair is presented to a subject, and where individually that subject has classed each of the two images as having different subjective quality, our subjects
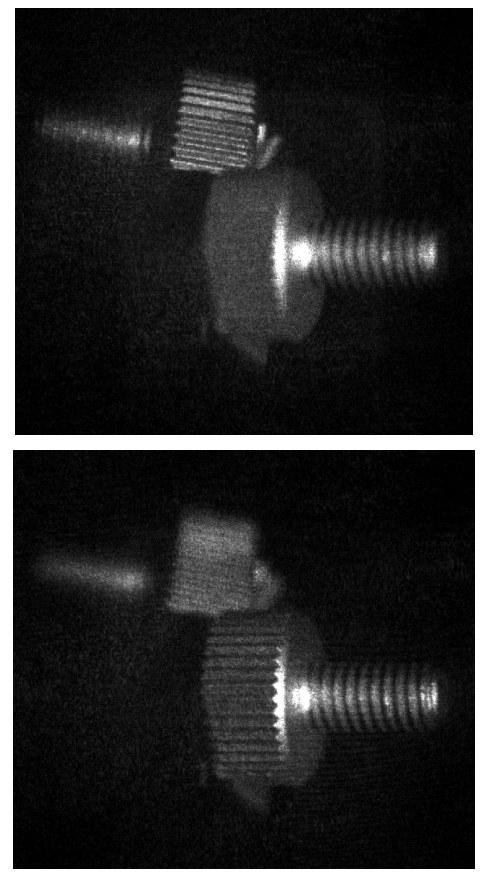

Figure 3: Disbalanced stereo pair: top, back of scene in focus; bottom, front of scene in focus.
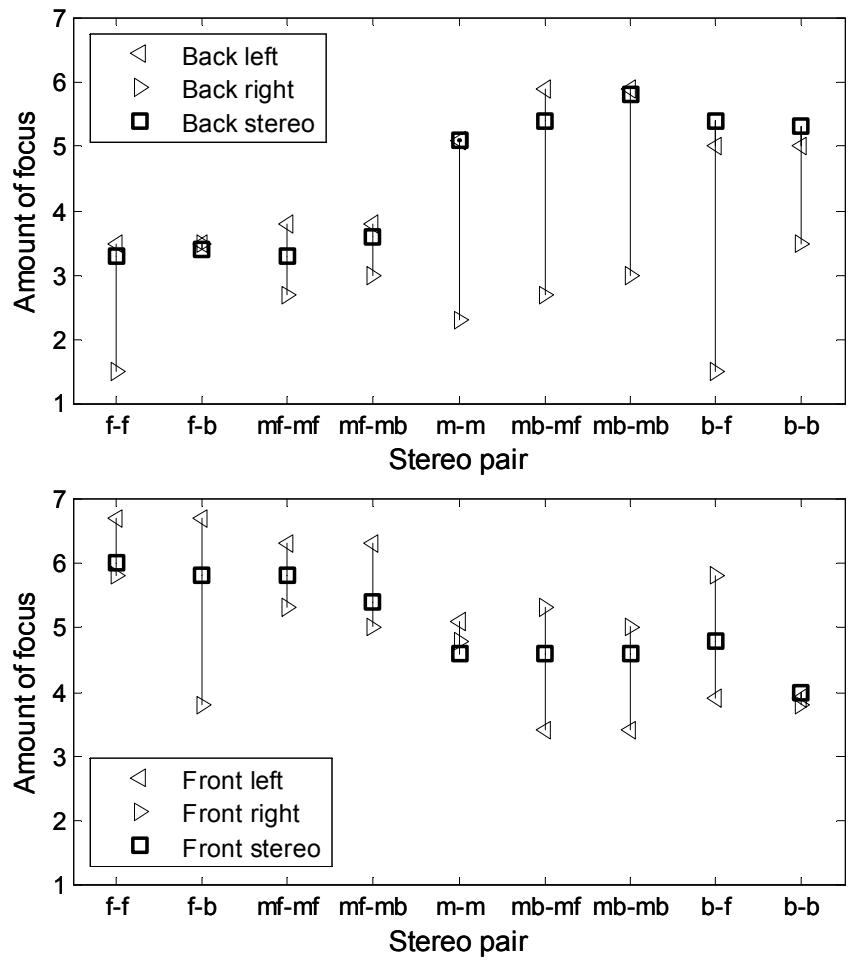

Figure 4: Amount of perceived focus in the back object (upper) and front object (lower) in the left and right individual reconstructions, and in the stereoscopically viewed pair, for each of nine stereo pairs. Average grades of all subjects plotted. Perceived focus in stereo is at least as good as the average of the in and out of focus versions. 
consistently rated the quality of the stereo pair as being as good as the better of the two images.

- In experiment 2, the stereo pairs were still perceived in 3D when one eye was presented with a reconstruction from the front of the scene and the other eye presented with a reconstruction from the back of the scene. However 2 out of 6 subjects consistently graded the $3 \mathrm{D}$ effect as being poor.

- The subjects' perceived amount of focus in individual reconstructions and in stereoscopically perceived image are shown in Fig. 4. When shown a disbalanced pair in stereo, subjects consistently perceived each object to be more in focus than its out-of-focus equivalent in individual reconstructions. Compared to an individual reconstruction where the back or front object was out of focus, the disbalanced stereo pair showed a mean increase in the back object of 1.8 and in the front object of 0.7. In both cases, the average was beaten.

\section{DISCUSSION}

There are implications for this work in terms of digital holographic video. If one uses a $2 \mathrm{D}$ monitor to display the intensity of a reconstruction from a digital hologram one needs to display a sequence of such $2 \mathrm{D}$ reconstructions to allow different perspectives to be viewed. To an observer this sequential visualization is perceived as rotation of the object. However, if movement is used to convey 3D information about the object then this approach is limited to viewing static 3D scenes. By using stereoscopic visualization we can retain this time dimension in digital holographic display. We can take a sequence of holograms of a moving object and in this way display digital holographic videos. Alternatively, the time dimension could be retained for some other purpose such as story telling.

\section{CONCLUSIONS}

In this paper we propose combining a stereoscopic display with digital holograms of real-world objects. We have demonstrated a way to visualize digital holographic data by using stereoscopic reconstruction pairs, and have studied aspects of 3D perception among a group of test subjects. In particular, we examined the effect of noise, poor quality, and out-of-focus reconstructions on 3D perception. We find that stereoscopic viewing, and our approach of presenting a different depth reconstruction to each eye, provides an efficient means of perceiving multiple in-focus planes of a hologram reconstruction volume with significantly reduced reconstruction computation time compared to extendedfocus image creation. Furthermore, in terms of also presenting different perspectives to each eye, we find that our approach more efficiently allows for $3 \mathrm{D}$ perception of objects encoded in digital holograms by not requiring an extended-focus perspective to be generated for each eye.

\section{ACKNOWLEDGEMENTS}

We thank Conor Mc Elhinney for capturing the digital holograms, and acknowledge support from Enterprise Ireland and Science Foundation Ireland.

\section{REFERENCES}

[1] D. Gabor, "A new microscopic principle," Nature, vol. 161, pp. 777-778, 1948.

[2] J.W. Goodman, R.W. Lawrence, "Digital image formation from electronically detected holograms," Applied Physics Letters, vol. 11, pp. 77-79, 1967.

[3] M.A. Kronod, N.S. Merzlyakov, L.P. Yaroslavskii, "Reconstruction of a hologram with a computer," Sov. Phys. Tech. Phys, vol. 17, p. 333, 1972.

[4] U. Schnars, W. Jueptner, Digital Holography: Digital Hologram Recording, Numerical Reconstruction, and Related Techniques, Springer, 2005.

[5] Th. Kreis, Handbook of Holographic Interferometry: Optical and Digital Methods, Wiley, 2005.

[6] L. Onural and P.D. Scott, "Digital decoding of in-line holograms," Optical Engineering, vol. 26, pp. 1124-1132, 1987.

[7] P. Ferraro, S. Grilli, D. Alfieri, S. De Nicola, A. Finizio, G. Pierattini, B. Javidi, G. Coppola, and V. Striano, "Extended focused image in microscopy by digital holography," Optics Express, vol. 13, 6738-6749, 2005.

[8] L. Onural and M.T. Ozgen, "Extraction of threedimensional object-location information directly from inline holograms using Wigner analysis," Journal of the Optical Society of America A, vol. 9, pp. 252-260, 1992.

[9] M. Sutkowski and M. Kujawinska, "Application of liquid crystal (LC) devices for optoelectronic reconstruction of digitally stored holograms," Optics and Lasers in Engineering, vol. 33, pp. 191-201, 2000.

[10] O. Matoba, T.J. Naughton, Y. Frauel, N. Bertaux and B. Javidi, "Real-time three-dimensional object reconstruction by use of a phase-encoded digital hologram," Applied Optics, vol. 41, pp. 6187-6192, 2002.

[11] J.C. Dainty, Laser Speckle and Related Phenomena, Springer Verlag, Berlin, second edition, 1984.

[12] G. Papelba, I. Cipane, and M. Ozolinsh, "Stereovision studies by disbalanced images," Advanced Optical Devices, Technologies, and Medical Applications, Proceedings of SPIE vol. 5123, pp. 323-329, 2003.

[13] L.M. Wilcox, J.H. Elder, R.F. Hess, "The effects of blur and size on monocular and stereoscopic localization," Vision Research, vol. 40, pp. 3575-3584, 2000.

[14] I. Yamaguchi, T. Zhang, "Phase-shifting digital holography," Optics Letters, vol. 22, pp. 1268-1270, 1997.

[15] Y. Frauel, E. Tajahuerce, M.A. Castro, B. Javidi, "Distortion-tolerant 3D object recognition using digital holography," Applied Optics, vol. 40, pp. 3887-3893, 2001. 